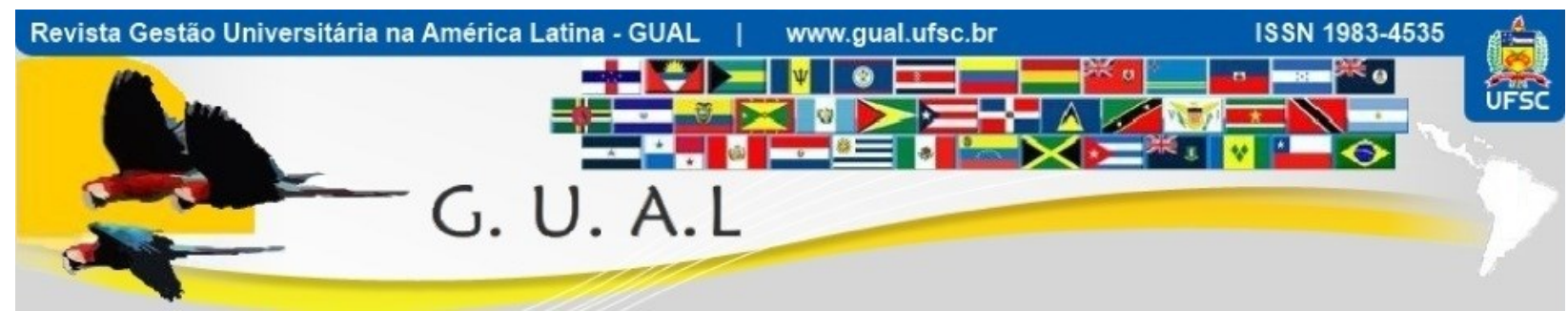

DOI: http://dx.doi.org/10.5007/1983-4535.2017v10n2p155

\title{
ANÁLISE METODOLÓGICA DAS DISSERTAÇÕES DEFENDIDAS NO PROGRAMA DE PÓS-GRADUAÇÃO EM ADMINISTRAÇÃO DA UCS
}

\author{
METHODOLOGICAL ANALYSIS OF DEFENDED DISSERTATIONS IN THE \\ GRADUATE PROGRAM IN BUSINESS MANAGEMENT AT UCS
}

Gabriela Zanandrea, Doutoranda Universidade de Caxias do Sul - UCS gabi.zanandrea@gmail.com

Tânia Craco, Doutoranda Instituto Federal do Rio Grande do Sul - IFRS tcraco@yahoo.com.br

Maria Emilia Camargo, Doutora Universidade de Caxias do Sul - UCS Kamargo@terra.com.br

Pelayo Munhoz Olea, Doutor Universidade de Caxias do Sul - UCS pelayo.olea@gmail.com

Uiliam Hahn Biegelmeyer, Doutorando Universidade de Caxias do Sul - UCS uiliam.hb@terra.com.br

Recebido em 15/dezembro/2015

Aprovado em 19/março/2017

Sistema de Avaliação: Double Blind Review 


\title{
RESUMO
}

O presente estudo compreende uma investigação sobre as práticas metodológicas de pesquisas em Administração. Para tanto teve por objetivo identificar as abordagens metodológicas adotadas nas dissertações do curso de Mestrado em Administração do Programa de Pósgraduação em Administração da Universidade de Caxias do Sul. Foi realizado um estudo exploratório/descritivo, com abordagem qualitativa, através da análise de conteúdo dos resumos e da metodologia dos trabalhos, categorizados de acordo com os métodos, abordagens, instrumentos e técnicas de pesquisa. Foram analisadas noventa e sete dissertações disponíveis on-line que permitiram delinear as características dos estudos elaborados no PPGA/UCS. Desse modo, das produções analisadas há predominância do enfoque qualitativo e da utilização de roteiro de entrevista como instrumento para coleta de dados. Assim, estes resultados demonstram que as pesquisas realizadas no Mestrado em Administração focam principalmente a compreensão aprofundada dos fenômenos investigados.

Palavras-chave: Produção científica. Dissertações. Mestrado em Administração. Abordagem metodológica.

\begin{abstract}
The present study comprises an investigation about the methodological research practices in Business Management. Therefore it has the goal to identify the methodological approaches taken in the dissertations of the Master's course of Business Management inside the Graduate Program in Business Management at the Caxias do Sul University. An exploratory/descriptive study was done, with qualitative approach, through the content analysis of the summaries and methodology of work, categorized according to the methods, approaches, tools and research techniques. Ninety seven on-line available dissertations were analyzed and allowed outline the characteristics of the studies prepared in PPGA / UCS. This way, from the analyzed productions there is the predominance of qualitative approach and the use of the interview guide as a tool for data collection. Thus, these results demonstrate that the research conducted in the Master in Business Management mainly focus on the comprehension depth in the investigated phenomena.
\end{abstract}

Key-words: Scientific production. Dissertations. Master in Business Administration. Methodological approach. 


\section{INTRODUÇÃO}

$\mathrm{O}$ avanço no desenvolvimento da ciência exige a abertura para a diversidade e interdisciplinaridade de conhecimentos, bem como, contextos que possibilitem as relações e interações, voltadas para a construção de saberes e práticas (HAMMERSCHMIDT et al., 2011). Nesse cenário, a Pós-Graduação (PG) se caracteriza como um ambiente que possibilita o desenvolvimento de pesquisadores, ações de pesquisa, processos de criação de conhecimentos e de novas tecnologias (HAMMERSCHMIDT et al., 2011). Cirani, Silva e Campanario (2012) complementam que a qualificação da Pós-Graduação e de seus discentes é essencial para o desenvolvimento científico, cultural, tecnológico e social de um país.

A Pós-Graduação stricto sensu no Brasil teve início em 1951, com a criação da CAPES (Coordenação de Aperfeiçoamento de Pessoal de Nível Superior), vinculada ao Ministério da Educação com o objetivo de executar a política nacional de Pós-Graduação (HAMMERSCHMIDT et al., 2011; CIRANI; SILVA; CAMPANARIO, 2012). Entretanto, a sua expansão ocorreu apenas em 1990 com o aumento da abrangência das suas áreas do conhecimento, diante da necessidade de formar docentes qualificados para atender à expansão do ensino superior no país, assim como, para expandir a capacidade investigativa das universidades através da formação de novos pesquisadores (HAMMERSCHMIDT et al., 2011).

A primeira pós-graduação Stricto Sensu em Administração no Brasil teve início em 1967, com o curso de mestrado em Administração, na Fundação Getúlio Vargas do Rio de Janeiro. Atualmente, na área de Administração estão em funcionamento no Brasil, 81 cursos de mestrado acadêmico, 46 de doutorado e 55 de mestrado profissional (CAPESb, 2014). Contudo, observa-se um número reduzido de pesquisas realizadas sobre o ensino Stricto Sensu da área de Administração (CIRANI; SILVA; CAMPANÁRIO, 2012).

Nesse sentido, destaca-se que os avanços das pesquisas propiciam a construção de novos conhecimentos que influenciam positivamente no desenvolvimento da área. Segundo Hair et al. (2003, p. 30), “a pesquisa é uma busca com o objetivo de discernir a verdade”. Para os autores, os reais pesquisadores estão sempre procurando por respostas, até mesmo no dia-adia quando se deparam com situações que exigem uma tomada de decisão (HAIR et al., 2003).

De acordo com Cruz e Ribeiro (2003) pesquisar significa buscar e compreender a forma como se processam os fenômenos observados, descrevendo sua estrutura e 
funcionamento. Sucintamente, a pesquisa científica tem o propósito de descobrir respostas para as questões propostas.

A produção científica se materializa por meio de defesas de dissertações e teses que são realizadas em diferentes contextos (SILVA, et al., 2013). Assim, diante do aumento das pesquisas em Administração através da produção de dissertações e teses faz-se necessário investigar as tendências, as abordagens metodológicas e teóricas destas produções, visando compreender melhor o crescimento nesta área (OPPA et al., 2012). Nesse sentido, o objetivo deste estudo foi identificar as abordagens metodológicas adotadas nas dissertações do curso de Mestrado em Administração do Programa de Pós-graduação em Administração da Universidade de Caxias do Sul.

\section{REFERENCIAL TEÓRICO}

\subsection{TIPOS DE PESQUISA}

Pesquisa é um procedimento intelectual, onde o pesquisador deve ter como objetivo adquirir conhecimento por meio da investigação de uma realidade e da constante busca por novas verdades sobre um fato (problema) (FACHIN, 2003). O resultado de uma pesquisa não é uma verdade absoluta, pois as descobertas são um processo inacabado e permanente) e toda a análise sobre um fato apresenta implicações de ordem apreciativa e analítica, cabendo ao pesquisador enquadrar no conhecimento empírico (MINAYO, 1993).

Ao se tratar de pesquisa, pode-se afirmar ainda que refere-se a uma atividade voltada à busca de respostas e à solução de problemas para questões propostas pelo pesquisador, através da utilização de métodos científicos, que são processos que visam aumentar a probabilidade de que a informação obtida seja significativa e precisa (SELLTIZ et al., 1974; CERVO; BERVIAN, 1996; LAKATOS; MARCONI (2003).

Sendo assim, Gil (2010) define a pesquisa social como o processo embasado em metodologias científicas que permite a obtenção de novos conhecimentos referente à realidade social. Neste contexto, verifica-se que há inúmeras classificações de pesquisa, apontadas por diversos autores, que serão apresentadas.

Gil (2010, p. 17), por sua vez, indica que "se pode definir pesquisa como o procedimento racional e sistemático que tem como objetivo proporcionar respostas aos problemas que são propostos". Essa conceituação remete à importância do desenvolvimento 


\section{ANÁLISE METODOLÓGICA DAS DISSERTAÇÕES DEFENDIDAS NO PROGRAMA DE PÓS- \\ GRADUAÇÃO EM ADMINISTRAÇÃO DA UCS \\ DOI: http://dx.doi.org/10.5007/1983-4535.2017v10n2p155}

cuidadoso do processo de pesquisa, que envolve inúmeras fases, desde a formulação do problema até a satisfatória apresentação dos resultados (GIL, 2010).

Considerando como pressuposto que a pesquisa é uma busca pela verdade, a pesquisa em administração, especificamente, é uma função de busca da verdade que reúne, analisa, interpreta e relata informações de forma que as decisões administrativas se tornem mais eficazes (HAIR et al., 2003).

Complementarmente, Cooper e Schindler (2001, p. 33) afirmam que "a pesquisa em administração é uma investigação sistemática que fornece informações para orientar as decisões empresariais". Assim, o valor da pesquisa em administração é inerente ao quanto que seus resultados auxiliam os gestores a tomarem melhores decisões (COOPER; SCHINDLER, 2001).

Desse modo, as pesquisas podem ser classificadas de acordo com diferentes taxonomias. Entretanto, ressalta-se que não há consenso na literatura quanto a estes delineamentos. Nesse sentido, este estudo aborda a taxonomia que classifica os estudos quanto à sua natureza, quanto aos objetivos, quanto à abordagem do problema e quanto aos procedimentos técnicos.

Quanto à natureza de pesquisa, os estudos podem ser classificados como básicos ou aplicados. Essa classificação considera a motivação do estudo (FERNANDES; GOMES, 2003). As pesquisas básicas, também denominadas de pesquisas puras, objetivam gerar conhecimentos novos úteis para o avanço da ciência sem aplicação prática prevista (LAKATOS; MARCONI, 2003), são motivadas pela curiosidade intelectual do pesquisador (VERGARA, 2000). Já as pesquisas aplicadas objetivam gerar conhecimentos para aplicação prática dirigida à solução de problemas específicos (GIL, 2010). Vergara (2000, p. 47) complementa que a pesquisa aplicada é "fundamentalmente motivada pela necessidade de resolver problemas concretos, mais imediatos ou não".

Quanto aos objetivos classificam-se como: descritivos, exploratórios ou explicativos. As pesquisas exploratórias visam proporcionar maior familiaridade e interação com o problema. Buscando reunir maior conhecimento sobre o fenômeno (GIL, 2010). Nesse sentido, Mattar (1999, p.80) afirma que "esse tipo de pesquisa é particularmente útil quando se tem uma noção muito vaga do problema de pesquisa". Cervo e Bervian (1996) complementam que os estudos exploratórios são normalmente o primeiro passo no processo de pesquisa, por propiciar maior experiência e informações sobre determinado assunto de 


\section{ANÁLISE METODOLÓGICA DAS DISSERTAÇÕES DEFENDIDAS NO PROGRAMA DE PÓS- \\ GRADUAÇÃO EM ADMINISTRAÇÃO DA UCS \\ DOI: http://dx.doi.org/10.5007/1983-4535.2017v10n2p155}

estudo. Destaca-se ainda que a formulação de hipóteses não é o elemento fundamental desta modalidade de pesquisa (CERVO; BERVIAN, 1996; FERNANDES; GOMES, 2003).

As pesquisas descritivas, por sua vez, descrevem as características de determinada população ou fenômeno ou então, o estabelecimento de relações entre as variáveis (LAKATOS; MARCONI, 2003). Este tipo de pesquisa responderá a questões como: quem, o quê, quando e onde (MATTAR, 1999). Dessa forma, essa modalidade é adotada quando o objetivo do trabalho for descrever as características de grupos, medir a proporção de elementos que possuam certas características ou comportamentos, dentro de uma população específica, desvendara ou verificar a existência de relação entre variáveis (MATTAR, 1999).

Por fim, as pesquisas explicativas, também conhecidas como causais (FERNANDES; GOMES, 2003), visam identificar os fatores que determinam ou contribuem para a ocorrência dos fenômenos. Além disso, aprofunda o conhecimento da realidade porque explica a razão, o "porquê" das coisas (GIL, 2010). Corroborando com este enforque Vergara (2000, p. 47) afirma que esse tipo de pesquisa "tem como principal objetivo tornar algo inteligível, justificar-lhe os motivos. Visa, portanto, esclarecer quais fatores contribuem, de alguma forma, para a ocorrência de determinado fenômeno."

Quanto aos procedimentos técnicos de acordo com Gil (2010) podem ser classificados como Pesquisa Bibliográfica; Pesquisa Documental; Pesquisa Experimental; Pesquisa Ex-Post Facto; Estudo de Coorte; Levantamento; Estudo de Campo; Estudo de Caso; Pesquisa Ação; Pesquisa Participante.

A pesquisa experimental consiste em determinar um objeto de estudo e selecionar as variáveis que seriam capazes de influenciá-lo. Nesse tipo de estudo, definem-se as formas de controle e de observação dos efeitos que a variável produz no objeto (GIL, 2010).

A pesquisa bibliográfica é aquela desenvolvida com base em material já elaborado, principalmente livros e artigos científicos, abordando a concepção de diversos autores, também podem ser utilizadas fontes de dados secundários (GIL, 2010). De acordo com Lakatos e Marconi (1996, p.66) tem por finalidade "colocar o pesquisador em contato direto com tudo o que foi dito, escrito ou filmado sobre determinado assunto". É caracterizada como uma das fontes mais importantes de pesquisa e compõe a fase prévia a ser feita em um processo de pesquisa, seja qual for o problema em questão (FERNANDES; GOMES, 2003).

$\mathrm{Na}$ pesquisa documental, a fonte da coleta de dados está restrita a documentos. Contudo, assemelha-se a bibliográfica, sendo que a diferença está na natureza das fontes, 
visto que a primeira utiliza materiais que não receberam ainda nenhum tratamento analítico (GIL, 2010). A vantagem deste tipo de pesquisa está diante do fato de utilizar documentos que constituem uma fonte rica e estável de dados (FERNANDES; GOMES, 2003). Contudo, sua principal desvantagem refere-se a não representatividade e a subjetividade dos documentos.

A pesquisa Ex-Post Facto, é aquela realizada a partir de fato passado, ou seja, o estudo é realizado após a ocorrência do fenômeno. Neste, o pesquisador não dispõe de controle sobre a variável que influência o fenômeno, pois ele já ocorreu (GIL, 2010).

$\mathrm{O}$ estudo de coorte refere-se àquele onde um grupo de pessoas que tem alguma característica comum, construindo uma amostra a ser acompanhada por certo período. Levantamento ou Survey refere-se aos estudos onde são realizadas interrogações diretas das pessoas cujo comportamento se deseja conhecer. Procura ser representativo de um universo definido (GIL, 2010).

No Estudo de caso é realizado um aprofundamento de um ou poucos objetos, explorando situações da vida real, além de descrever a situação do contexto em que está sendo feita determinada investigação. Nesse sentido, busca explicar variáveis de determinado fenômeno (GIL, 2010, p.32). Yin complementa que estudo de caso "é uma investigação empírica que investiga um fenômeno contemporâneo dentro de seu contexto da vida real, especialmente quando os limites entre o fenômeno e o contexto não são claramente definidos" (YIN, 2005, p.32).

$\mathrm{Na}$ pesquisa-ação há o envolvimento ativo do pesquisador, ocorre à intervenção participativa na realidade social, sendo intervencionista. $O$ enfoque da pesquisa-ação requer que o pesquisador interprete o mundo real a partir da perspectiva dos sujeitos de sua investigação (ROESCH, 1996).

Pode-se ainda classificar as pesquisas quanto à abordagem do problema: qualitativa, quantitativa ou mista. A pesquisa qualitativa é uma série de técnicas interpretativas que procuram descrever, decodificar, traduzir e, de alguma forma, chegar a um acordo com o significado, não a frequência, de certos fenômenos que ocorrem de forma mais ou menos natural no mundo social (EASTERBY-SMITH; THORPE; LOWE, 1999). Complementam Sampieri, Collado e Lucio (2006), que esse tipo de pesquisa, emprega a coleta de dados sem medição numérica para desvendar ou aprimorar questões de pesquisa.

Já a pesquisa quantitativa emprega a coleta e a análise de dados a fim de responder às questões de pesquisa e testar hipóteses previamente estabelecidas. Além disso, confia na 
medição numérica, na contagem e frequentemente no uso de estatística para estabelecer com exatidão os padrões de comportamento de uma população (SAMPIERI; COLLADO; LUCIO, 2006). A pesquisa também pode ser classificada como mista quando reúne tanto dados quantitativos e qualitativos (GIL, 2010).

\section{METODOLOGIA}

O presente estudo se caracteriza quanto à natureza como uma pesquisa básica, quanto à abordagem do problema como mista, quanto aos objetivos como descritiva e quanto aos procedimentos técnicos como documental (GIL, 2010).

De acordo com o objetivo desta pesquisa foi realizada uma análise das dissertações do PPGA/UCS, defendidas no período de 2008 a 2012. A lista das dissertações do programa com os respectivos títulos defendidos em cada ano e o orientador foi obtida através dos Cadernos de Indicadores (Teses e dissertações) da Avaliação Trienal da CAPES (CAPES, 2014a).

A caracterização das dissertações foi realizada por meio da leitura do resumo e dos capítulos e sub-capítulos referentes à metodologia. Os dados coletados foram categorizados em uma ordenação preliminar de acordo com a abordagem metodológica utilizada, classificada nos seguintes elementos: número identificador; ano de defesa; linha de pesquisa; quanto à natureza de pesquisa: básica ou aplicada; abordagem do problema: qualitativa, quantitativa ou mista; objetivos: exploratórias, descritivas e explicativas; procedimentos técnicos, técnicas de coleta de dados, técnicas de análise dos dados e softwares utilizados.

Os dados foram coletados com base nas dissertações disponíveis na página da Universidade de Caxias do Sul, e posteriormente processados em planilhas do Microsoft Excel. Para a descrição das frequências foi utilizado o programa estatístico SPSS (Statistical Package for Social Sciences).

\section{AMBIÊNCIA DE PESQUISA}

O Programa de Pós-graduação em Administração da Universidade de Caxias do Sul (PPGA-UCS) está vinculado à Pró-Reitoria de Pesquisa e Pós-Graduação e foi implantado em 2006 com o curso de Mestrado, cujo objetivo tem como principal característica "estabelecer um equilíbrio perfeito entre o mundo acadêmico e o mundo empresarial, interagindo com o setor público e privado, na geração e difusão de conhecimentos relevantes e aplicados ao seu contexto" (UCS, 2014). Posteriormente em 2010 foi incorporado o curso de Doutorado em 
Administração em Associação Ampla com a Pontifícia Universidade Católica do Rio Grande do Sul (CAPES, 2014c).

Um aspecto a ser apontado são a área de concentração e as linhas de pesquisa do Programa. Área de concentração refere-se a delimitação das fronteiras do campo do conhecimento da investigação (MENANDRO, 2003). Enquanto linhas de pesquisa são definidas como os troncos de onde procedem as disciplinas e a produção científica do programa (PETERSEN, 2006). Linhas de pesquisas também podem ser entendidas de acordo com o CNPq (2010) como temas aglutinadores de estudos científicos que se fundamentam em tradição investigativa, de onde se originam projetos cujos resultados guardam afinidades entre si. Ou seja, as provem das experiências e pesquisas realizadas pelos docentes.

Nesse sentido, o curso de Mestrado concentra-se na área de Administração da Produção, ancorado em duas linhas de pesquisa: Estratégia e Gestão da Produção e Gestão da Inovação e Competitividade. A linha Estratégia e Gestão da Produção contempla pesquisas voltadas para estratégias de produção empregadas por empresas da região da Serra Gaúcha, área em que está inserida a Universidade de Caxias do Sul. A linha Gestão da Inovação e Competitividade abrange estudos sobre as dimensões relacionadas com a gestão da inovação e a competitividade como fonte de crescimento, em um contexto global, das empresas da região da Serra Gaúcha.

No ano de 2014 o referido programa contava com 13 professores permanentes e 1 colaborador (docentes colaboradores são aqueles com atuação sistemática no programa que não sejam servidores públicos ativos ou celetistas vinculados à respectiva IES) (CAPES, 2014c). Desde a sua criação o Mestrado em Administração passou por duas avaliações trienais da CAPES. Na primeira avaliação no triênio de 2007-2009 recebeu nota 3, evoluindo para o triênio seguinte (2010-2012) para nota 4 (CAPES, 2014a).

No ano de 2013, o PPGAUCS passou a publicar a Revista Brasileira de Gestão e Inovação com periodicidade quadrimestral está voltada para reflexões e estudos na ciência da administração.

\section{RESULTADOS}

A pesquisa foi realizada no período de 2008 a 2012, onde foram analisadas as dissertações defendidas no Programa de Pós-Graduação em Administração da Universidade de Caxias do Sul (PPGA/UCS), disponíveis na Biblioteca Digital Brasileira de Teses e 
Dissertações (BDTD) da biblioteca da UCS. A partir da lista obtida junto à CAPES observou-se que foram defendidas no período analisado 101 trabalhos, entretanto, considerouse neste estudo apenas aquelas disponíveis online, desse modo, 4 estudos foram excluídos do portfólio, restando 97 que foram posteriormente analisados.

Na Tabela 1 está destacado o número de dissertações defendidas no período estudado.

Tabela 1 Ano das dissertações.

\begin{tabular}{c|c|c}
\hline Ano & Frequência & \% \\
\hline 2008 & 22 & 22,7 \\
2009 & 21 & 21,6 \\
2010 & 19 & 19,6 \\
2011 & 16 & 16,5 \\
2012 & 19 & 19,6 \\
Total & 97 & 100 \\
\hline
\end{tabular}

Fonte: Elaborado pelos autores (2015).

Pode-se observar que no ano de 2008 foram defendidas 22 dissertações, 2009 foram 21, 2010 foram defendidas 19 dissertações, em 2011 foram 16 e por fim em 2012 foram 19 trabalhos de conclusão defendidos.

Foi verificado também a distribuição por linha de pesquisa e por ano, observa-se na Tabela 2 que a linha de Gestão da Inovação e Competitividade apresentou 54 trabalhos enquanto que a linha Estratégia e Gestão da Produção apresentou 43 trabalhos. Considerando as diferenças entre as linhas por ano, observa-se que o ano em que houve maior diferença na quantidade de trabalhos apresentados foi em 2009 onde a Linha voltada para inovação e competitividade teve 7 trabalhos a mais que de gestão da produção.

Tabela 2 Linha de pesquisa das dissertações.

\begin{tabular}{l|c|c|c|c|c|c}
\hline \multirow{2}{*}{ Linha } & \multicolumn{7}{|c}{ Ano } & $\mathbf{2 0 1 1}$ & Total \\
\cline { 2 - 7 } & $\mathbf{2 0 0 8}$ & $\mathbf{2 0 0 9}$ & $\mathbf{2 0 1 0}$ & $\mathbf{2 0 1 1}$ & $\mathbf{2 0 1 2}$ & 43 \\
\hline $\begin{array}{l}\text { Estratégia e gestão da produção } \\
\text { Gestão da inovação }\end{array}$ & 10 & 9 & 12 & 6 & 6 & 54 \\
competitividade & 12 & 12 & 7 & 10 & 13 & \\
Total & 22 & 21 & 19 & 16 & 19 & 97 \\
\hline
\end{tabular}

Fonte: Elaborado pelos autores (2015).

Com a finalidade de organizar e estruturar o presente trabalho, será analisado sete categorias: abordagem, natureza, objetivos, procedimentos, instrumentos de análise, análise dos dados e softwares utilizados na pesquisa. 
Diante do levantamento e consulta dos dados das dissertações, apresentados na Tabela 3 observou-se que 53,6\% apresentaram abordagem qualitativa, 17,5\% abordagem mista, 27,8 $\%$ abordagem quantitativa e em 1 dos trabalhos analisados não estava descrito qual a abordagem de pesquisa utilizada.

Tabela 3 Abordagem da pesquisa.

\begin{tabular}{l|c|c}
\hline Abordagem & Frequência & \% \\
\hline Qualitativa & 52 & 53,6 \\
Quantitativa & 17 & 17,5 \\
Mista & 27 & 27,8 \\
Não especificado & 1 & 1,0 \\
Total & 97 & 100,0 \\
\hline
\end{tabular}

Fonte: Elaborado pelos autores (2015).

$\mathrm{Na}$ Tabela 4 destaca-se a natureza da pesquisa dos estudos, constata-se que $83,5 \%$ dos trabalhos consultados não especificaram qual o tipo de natureza adotada pelo estudo, enquanto $14,4 \%$ foram classificadas como pesquisa aplicada e 2,1\% como pesquisa básica.

Tabela 4 Natureza da pesquisa.

\begin{tabular}{l|c|c}
\hline Natureza & Frequência & $\mathbf{\%}$ \\
\hline Básica & 2 & 2,1 \\
Aplicada & 14 & 14,4 \\
Não especificada & 81 & 83,5 \\
Total & 97 & 100,0 \\
\hline
\end{tabular}

Fonte: Elaborado pelos autores (2015).

Considerando os objetivos levantados, conforme demonstrado na Tabela 5, constatouse a predominância de trabalhos com cunho objetivos exploratórios com 46,4\%, exploratório e descritivos $30,9 \%$, objetivo descritivo $13,4 \%$, explicativos $1,0 \%$, e não especificados $8,2 \%$.

Tabela 5 Objetivos da pesquisa.

\begin{tabular}{l|c|c}
\hline Quanto aos objetivos & Frequência & \% \\
\hline Exploratória & 45 & 46,4 \\
Descritiva & 13 & 13,4 \\
Explicativa & 1 & 1,0 \\
Exploratória e Descritiva & 30 & 30,9 \\
Não especificada & 8 & 8,2 \\
Total & 97 & 100,0 \\
\hline
\end{tabular}

Fonte: Elaborado pelos autores (2015). 
Ressalta-se que quanto aos procedimentos técnicos utilizados, podem-se observar na Tabela 6, uma predominância de estudo de caso em 45,4\% dos trabalhos, seguido pela survey/levantamento em $26,8 \%$; pesquisa documental em $25,8 \%$; estudo de caso múltiplos em 14,4\%; grupos focais em 3,1\%; pesquisa experimental e pesquisa ação em $1,0 \%$ e por fim, $7,2 \%$ dos estudos não especificaram os procedimentos técnicos.

Tabela 6 Procedimentos da pesquisa.

\begin{tabular}{l|c|c}
\hline Quanto aos procedimentos & Frequência & \% \\
\hline Pesquisa documental & 25 & 25,8 \\
Pesquisa Experimental & 1 & 1,0 \\
Survey & 26 & 26,8 \\
Pesquisa ação & 1 & 1,0 \\
Estudo de caso & 44 & 45,4 \\
Grupos focais & 3 & 3,1 \\
Estudo de caso múltiplo & 14 & 14,4 \\
Estudo de campo & 5 & 5,2 \\
Não especificada & 7 & 7,2 \\
\hline
\end{tabular}

Fonte: Elaborado pelos autores (2015).

$\mathrm{Na}$ Tabela 7 foram expostos os instrumentos de coletas usados nas pesquisas, prevalecendo o roteiro de entrevista com 44,3\%, seguido pelo questionário com $29,9 \%$, roteiro e questionário com $15,5 \%$, roteiro e observação com $6,2 \%$, outros instrumentos $1,0 \%$ e não especificado com $3,1 \%$.

Tabela 7 Instrumentos para coleta de dados da pesquisa.

\begin{tabular}{l|c|c}
\hline Instrumentos para coleta de dados & Frequência & \% \\
\hline Roteiro de entrevista & 43 & 44,3 \\
Questionário & 29 & 29,9 \\
Roteiro e questionário & 15 & 15,5 \\
Roteiro e observação & 6 & 6,2 \\
Outros instrumentos & 1 & 1,0 \\
Não especificada & 3 & 3,1 \\
Total & 54 & 100 \\
\hline
\end{tabular}

Fonte: Elaborado pelos autores (2015).

Já na Tabela 8 está demonstrado a forma como os dados foram analisados, observa-se que prevaleceu análise de conteúdo com 57,7\%, estatística descritiva com 30,9\%, análise fatorial com 23,7\%, análise de variância 20,6\%, análise de regressão 15,5\%, análise de 
correlação $18,6 \%$, construção de cenários 5,2\%, estatística inferencial com 4,0\%, modelagem de equações estruturais com $1,0 \%$ e não especificada com $6,2 \%$.

Tabela 8 Análise dos dados da pesquisa.

\begin{tabular}{l|c|c}
\hline Análise dos dados & Frequência & \% \\
\hline Análise de conteúdo & 56 & 57,7 \\
Estatística descritiva & 30 & 30,9 \\
Análise fatorial & 23 & 23,7 \\
Análise de regressão & 15 & 15,5 \\
Análise de variância & 20 & 20,6 \\
Análise de correlação & 18 & 18,6 \\
Construção de cenários & 5 & 5,2 \\
Estatística inferencial & 4 & 4,1 \\
Modelagem de Equações Estruturais & 1 & 1,0 \\
Não especificada & 6 & 6,2 \\
\hline
\end{tabular}

Fonte: Elaborado pelos autores (2015).

Na Tabela 9 pode-se observar os softwares utilizados nas pesquisas. Destaca-se que a maioria dos estudos $(52,6 \%)$ não especifica o software que utilizou para análise dos dados coletados. Contudo, dentre os especificados, destaca-se que a maioria dos estudos utilizou o Programa estatístico SPSS com 33,0\%, Microsoft Excel 16,5\%, Puma 4,1\% e Bioestat, Atlas TI, NVivo, SQDA, AMOS todos com 1,0\%.

Tabela 9 Softwares usados na pesquisa.

\begin{tabular}{l|c|c}
\hline Software & Frequência & \% \\
\hline SPSS & 32 & 33,0 \\
Bioestat & 1 & 1,0 \\
Microsoft Excel & 16 & 16,5 \\
Puma & 4 & 4,1 \\
Atlas Ti & 1 & 1,0 \\
NVivo & 1 & 1,0 \\
SQDA & 1 & 1,0 \\
AMOS & 1 & 1,0 \\
Sphinx & 1 & 1,0 \\
Não especificada & 51 & 52,6 \\
\hline
\end{tabular}

Fonte: Elaborado pelos autores (2015).

\section{CONCLUSÃO}

Ao analisar as publicações do PPGA-UCS, considerando as características analisadas no presente estudo, constatou-se que os trabalhos desenvolvidos apresentam forma e conteúdo diversificados, porém, há uma predominância de trabalhos exploratórios com abordagem 
qualitativa. Este resultado demonstra que os trabalhos realizados buscam estabelecer um aprofundamento na compreensão da natureza do fenômeno investigado. Em detrimento de pesquisas voltadas para quantificação de variáveis, associações entre elas ou de mensuração destas relações.

Outro aspecto relevante refere-se a número de trabalhos que utilizaram abordagem mista, ou seja, concomitantemente qualitativa e quantitativa. Este resultado denota o detrimento de possibilidades de se realizar pesquisas estabelecendo a associações entre variáveis através da abordagem quantitativa ao mesmo tempo em que interpretasse os seus significados através da abordagem qualitativa.

Quanto à natureza de pesquisa houve a prevalência de estudos aplicados, este resultado pode estar relacionado com o fato de que as pesquisas na Área de Administração busquem principalmente a soluções de problemas e a aplicação dos conhecimento e resultados obtidos, o que vai ao encontro das características deste tipo de pesquisa.

Quanto aos procedimentos técnicos a maioria utilizou estudos de caso, cuja principal forma de coleta de dados foi os roteiros de entrevista e na análise dos dados prevaleceu a análise de conteúdo. Estes resultados demonstram a coerência entre as abordagens, procedimentos técnicos e técnicas de coleta adotadas nas dissertações.

Sob estas condições, destaca-se a possibilidade de diversificar as metodologias utilizadas nas pesquisas do PPGA-UCS, através do aumento de estudos com abordagem quantitativa ou que adotem ambas as abordagens, quali-quantitativa, bem como, utilizando outras técnicas de investigação, assim, auxiliando na evolução das pesquisas do referido programa.

Destaca-se também que após a análise dos trabalhos pode-se constar que as metodologias geralmente eram pouco detalhadas, e que não há uma similaridade na estrutura utilizada para elaboração da metodologia utilizada no estudo. Posteriori, sugere-se uma padronização dos procedimentos metodológicos, possibilitando dessa forma uma explicitação das trajetórias seguidas pelos acadêmicos, isso possibilitaria uma ampliação das probabilidades de replicação dessa pesquisa.

Para estudos futuros, recomenda-se a realização de pesquisas com o intuito de identificar as tendências temáticas predominantes no PPGA-UCS, e a relação com as linhas de pesquisa do curso e com os temas abordados nos artigos publicados no mesmo período. 
Assim, destaca-se a importância da qualidade das pesquisas científicas na área de Administração, com o aumento das exigências quanto ao rigor metodológico, certificando uma perspectiva mais científica para os trabalhos realizados.

\section{REFERÊNCIAS}

CAPES - Coordenação de Aperfeiçoamento de Pessoal de Nível Superior. Proposta do Programa. Caderno de Indicadores. 2014a. Disponível em:

http://conteudoweb.capes.gov.br/conteudoweb/VisualizadorServlet?nome=/2012/42008018/0 27/2012_027_42008018007P8_Proposta.pdf\&aplicacao=projetoRelacaoCurso. Acesso em 01 de julho de 2014 .

. Mestrado e doutorados reconhecidos. 2014b. Disponível em https://sucupira.capes.gov.br/sucupira/public/docs/manual_coleta.pdf. Acesso em 01 de julho de 2014.

Coleta de Dados: Conceitos e orientações. 2014c. Disponível em https://sucupira.capes.gov.br/sucupira/public/docs/manual_coleta.pdf. Acesso em 01 de julho de 2014.

CERVO, A. L.; BERVIAN, P. A. Metodologia científica. 4ª ed. São Paulo: Makron Books, 1996.

CIRANI, C. B. S.; SILVA, H. H. M.; CAMPANARIO, M. A. A evolução do ensino da pósgraduação estrito senso em administração no Brasil. Rev. adm. contemp. Curitiba, v. 16, n. 6, Dec. 2012

CNPq. Conselho Nacional de Desenvolvimento Científico e Tecnológico (CNPq). Diretório dos Grupos de Pesquisa [Internet]. 2010

COOPER, D. R.; SCHINDLER, P. S. Métodos de Pesquisa em Administração. São Paulo: Bookman, 2001.

CRUZ, C.; RIBEIRO, U. Metodologia Científica: teoria e prática. Rio de Janeiro: Axcel Books, 2003.

DEMO, P. Pesquisa e construção de conhecimento. Rio de Janeiro: Tempo Brasileiro, 1996.

EASTERBY-SMITH, M.; THORPE, R.; LOWE, A. Pesquisa gerencial em administração: um guia para monografias, dissertações, pesquisas internas e trabalhos em consultoria. São Paulo: Pioneira, 1999. 172 p.

FACHIN, O. Fundamentos de metodologia. 4. ed. São Paulo: Saraiva, 2003.

FERNANDES, L.A.; GOMES, J.M.M. Relatórios de pesquisa nas ciências sociais: características e modalidades de investigação. Contexto, Porto Alegre, v. 3, n. 4, 2003. 
GIL, A. C. Como elaborar projetos de pesquisa. 5. ed. São Paulo: Atlas, 2010.

HAIR, J. F., Jr.; BABIN, B.; MONEY, A. H.; SAMOUEL, P. Fundamentos de Métodos de Pesquisa em Administração. São Paulo: Bookman, 2003.

HAMMERSCHMIDT, Karina Silveira de Almeida et al. Dissertações produzidas no Programa de Pós-Graduação e Mestrado em Enfermagem da Universidade Federal do Paraná. Rev. esc. enferm. USP, São Paulo, v. 45, n. 6, Dec. 2011.

LAKATOS, E. M.; MARCONI, M. A. Fundamentos de metodologia científica. 5.ed. São Paulo: Atlas, 2003.

MATTAR, F. N. Pesquisa de marketing: metodologia, planejamento. 5. ed. São Paulo: Atlas, 1999. $1 \mathrm{v}$.

MENANDRO, P. R. M. Linha de pesquisa: possibilidades de definição e tipos de utilização do conceito. Rev. adm. contemp. Curitiba, v. 7, n. 2, 2003.

MINAYO, M. C. S. \& SANCHES, O. Quantitativo-Qualitativo: Oposição ou Complementaridade? Cad. Saúde Públ., Rio de Janeiro, 9 (3): 239-262, jul/set, 1993.

PETERSEN, S.R.F. A Pós-Graduação em história: novas e velhas questões. Anos 90, Porto Alegre, v. 13, n. 23/24, p.29-44, jan./dez. 2006.

ROESCH, S. M. A. Projetos de estágio e de pesquisa em administração: guia para estágios, trabalhos de conclusão, dissertações e estudo de caso. 2. ed. São Paulo: Atlas, 1999.

OPPA, D. F. et al. Tipos de pesquisa utilizados na área de atividade física e saúde do Programa de Pós-Graduação em Educação Física da Universidade Federal de Santa Catarina. EFDeportes.com, Revista Digital. Buenos Aires, Ano 16, nº. 164, 2012.

SAMPIERI, R.; COLLADO, C.; LUCIO, P. Metodologia de Pesquisa. São Paulo: McGrawHill, 2006.

SELLTIZ, C.; WRIGHTSMAN, L.; COOK, S.; KIDDEER, L. Métodos de Pesquisa nas Relações Sociais. São Paulo, EPV-EDUSP, 1974.

SILVA, et al. Análise metodológica da produção acadêmica no programa de pós-graduação em administração da UFRN. Tekhne e Logos, Botucatu, SP, v.4, n.2, 2013.

UCS - Universidade de Caxias do Sul. Programa de Pós-Graduação em Administração. Disponível em http://www.ucs.br/site/pos-graduacao/formacao-stricto-sensu/administracao/ Acesso em 01 de julho de 2013.

VERGARA, S. C. Projetos e relatórios de pesquisa em administração. 3. ed. São Paulo: Atlas, 2000.

YIN, R. K. Estudo de Caso: planejamento e métodos. 3.ed. Porto Alegre: Bookman, 2005. $212 \mathrm{p}$. 